\title{
Spreadsheet-Based Program for Alignment of Overlapping DNA Sequences
}

BioTechniques 26:1180-1185 (June 1999)

\author{
Ramaswamy Anbazhagan and Edward \\ Gabrielson \\ Johns Hopkins University School of Medicine, \\ Baltimore, MD, USA
}

workbook specifically designed for this and is capable of identifying overlapping regions between two sequence fragments and displaying the sequence alignment.

\section{MATERIALS AND METHODS}

\section{Downloading and Opening the Program}

\begin{abstract}
Molecular biology laboratories frequently face the challenge of aligning small overlapping DNA sequences derived from a long DNA segment. Here, we present a short program that can be used to adapt Excel ${ }^{\circledR}$ spreadsheets as a tool for aligning DNA sequences, regardless of their orientation. The program runs on any Windows ${ }^{\circledR}$ or Macintosh ${ }^{\circledR}$ operating system computer with Excel 97 or Excel 98. The program is available for use as an Excel file, which can be downloaded from the BioTechniques Web site. Upon execution, the program opens a specially designed customized workbook and is capable of identifying overlapping regions between two sequence fragments and displaying the sequence alignment. It also performs a number of specialized functions such as recognition of restriction enzyme cutting sites and $C p G$ island mapping without costly specialized software.
\end{abstract}

\section{INTRODUCTION}

Sequencing large DNA segments typically requires cutting the DNA into a number of small overlapping fragments, which are then individually cloned and sequenced from both ends. These fragment sequences can be in forward, reverse, complimentary or antiparallel orientations. Identification of the overlapping segments between various fragments and arranging them into a single continuous sequence is a time-consuming process. Furthermore, software available commercially for sequence assembly is relatively expensive. Here, we report the adaptation of a commonly available spreadsheet program, Excel ${ }^{\circledR} 97$ or Excel 98 (Microsoft, Redmond, WA, USA) for this purpose. The program opens a customized
We have created an Excel file with a built-in program that performs all major functions required for sequence alignment. The program works well with Excel 97 for Windows ${ }^{\circledR}$ operating system and with the Excel 98 for Macintosh ${ }^{\circledR}$ (Apple Computer, Cupertino, CA, USA) operating system. We recommend downloading the file (alignwin.xls for Windows and alignmac.xls for Macintosh operating system) from the Software Library on the BioTechniques Web site (http:// www.BioTechniques.com). The program can be started in two ways: (i) either click open the downloaded file or (ii) start the Excel program and open the downloaded file from within the program using File/Open. A dialogue box appears asking to enable or disable macros. Click on the Enable macros button. When the program opens, you will notice that a new menu named Align is added to the program menu bar. This menu has six menu items, namely New-Workbook, Convert, Paste, Mark-Align, Single-Step and Clear-Sheet2. These menu items can be executed to open a customized workbook and to perform various functions necessary to identify the region of alignment between two overlapping sequences.

\section{Opening a New Workbook and Entering a Sequence}

The Excel workbook is organized as a collection of sheets, with each sheet's name appearing on a tab at the bottom of the workbook. Navigation between the sheets is possible by clicking on the tab at the bottom. Refer to the User's Guide for further explanation about the organization and use of Excel workbook and sheets. Go to the Align menu and click on the New-Workbook menu item. A new workbook is opened with three sheets. The program automatically enters the col- 
umn headings such as Clone ID, Primer, Count and Sequence in sheet 1, as shown in Figure 1. The user enters the relevant details of the sequence fragments in the corresponding columns manually. The clone identification numbers are entered in column A, and the sequencing primers such as T7, T3 and M13 are entered in column B. If a new insert-specific oligonucleotide is used as a primer for sequencing, the sequence of the primer itself can be entered in column B. The sequences read from the sequencing gel are entered in column $\mathrm{D}$, using the standard abbreviation (a, c, g and t). Ambiguous nucleotides should be designated as "n". Each cell in Excel 97 can hold up to 32767 characters, and therefore, very long sequences can be entered in these cells.

The program automatically fills up column $\mathrm{C}$ with a formula that will count and display the total number of nucleotides of the sequences that are entered in column $\mathrm{D}$ by the user. Column $\mathrm{C}$ initially displays 0 before the entry of any sequence in column D. When the sequences are entered or copied to column D, the nucleotide count of each sequence is automatically displayed in column C. Enter the sequences using lowercase letters. The program codes are case-sensitive and will function properly only if the sequences are entered in lowercase letters. The nucleotide count in column $\mathrm{C}$ is updated automatically whenever any sequence entry is altered in column D.

The second sheet serves as a temporary worksheet for running the macros to align any two sequences. The labels Clone 1, Clone 2 and Overlap are automatically displayed by the program in sheet 2 in the appropriate cells. The overlapping fragments, identified by the program, are arranged manually into a single continuous sequence in the third sheet, which is customized by the program for this purpose.

\section{Sequence Alignment}

To align the sequences, it is first necessary to search for the overlapping sequences between different clones. During cloning, the fragments can get oriented in any direction, so that the overlapping sequences can be matching in forward, reverse, compliment or antiparallel orientations. Thus, it is necessary to search and align all possible orientations of the fragment sequences. The entire alignment can be performed as a single step or as a series of three steps. We first present the three-step approach to illustrate the strategy used and to demonstrate additional options available.

Select two sequences from sheet 1 to be aligned, and copy them into cells B1 and B3 of sheet 2. Click on the Align menu, and click on the Convert menu item; this will convert the sequence in cell $\mathrm{B} 1$ into a reverse sequence, which is pasted in cell B2. The second sequence pasted in cell B3 is converted into reverse, complimentary and antiparallel orientations and pasted into cells B4, B5 and B6, respectively. The program also finds the longest overlapping region between these two groups of sequences and displays the overlapping sequences in cells B9 and B10. The program is capable of converting and aligning sequences of any length entered in the appropriate cells. The program also can handle ambiguous nucleotides. If the sequence contains an " $n$ ", indicating ambiguity, it will be aligned against any of the four nucleotides. If the overlapping region contains any ambiguous nucleotide, then the sequence in B9 and B10 reflects this.

Next, execute the menu item Paste, which will paste all sequences in cells B1 through B6 in a column format (starting from cell B13) with each nucleotide occupying a single cell. Executing the menu item Mark-Align is the next step; this reads the overlapping sequence from cells B9 and B10 and uses them as search strings to search the vertically pasted sequences, and the overlapping regions are highlighted with a bright pink background. The actual alignment of the two sequences is also displayed in column J, with the overlapping regions highlighted. Figure 2 shows a sample sheet 2 displaying the results of all three procedures. Sequence alignments can be repeated for different sets of sequences. To erase the previous entries, including the alignment, click on the ClearSheet2 menu item in the Align menu.

If there is a true overlapping region between the two sequences, the program always identifies that. In most other cases, the program identifies a couple of nucleotides or a single nucleotide at the terminal region as the region of alignment. It is up to the user to decide whether these few nucleotide overlaps reflect real alignment or not. For example, if you are sequencing a known insert with multiple primers, and if you know the position and sequence of the primers used, then you will be able to identify and confirm even if there is an overlap of a few nucleotides as a valid result. If you are sequencing a novel insert, and if you get an overlap of a few nucleotides between two fragments, you might need further sequencing data from different primers to confirm or exclude this result.

In some instances, when the Mark-Align menu item is executed, the program will identify and mark more than one region in each sequence as the region of alignment. This might

\begin{tabular}{|l|c|c|l|l|}
\hline Clone ID & Primer & Count & Sequence \\
\hline Clone 1 & T3 & 53 & acagagatagacagatacagctcgetgacagatcgetccagtacgctgacacg \\
\hline Clone 2 & T7 & 50 & atatatagacacagaacagacagagatagatagctttgcctcgccgttta \\
\hline Clone 3 & M13 & 54 & tacgctgacacgagatagcttcgctcgctcgaagatgcctcgacagtcccet \\
\hline Clone 4 & T3 & 51 & tcgaagatgcctcgacagtcgccatctcgctacccacatatagaccatgaa \\
\hline
\end{tabular}

Figure 1. First sheet (sheet 1) of the Excel workbook. Clone identification numbers, primers and sequence information are shown. 
Quebecor - place LHP BioComputing head

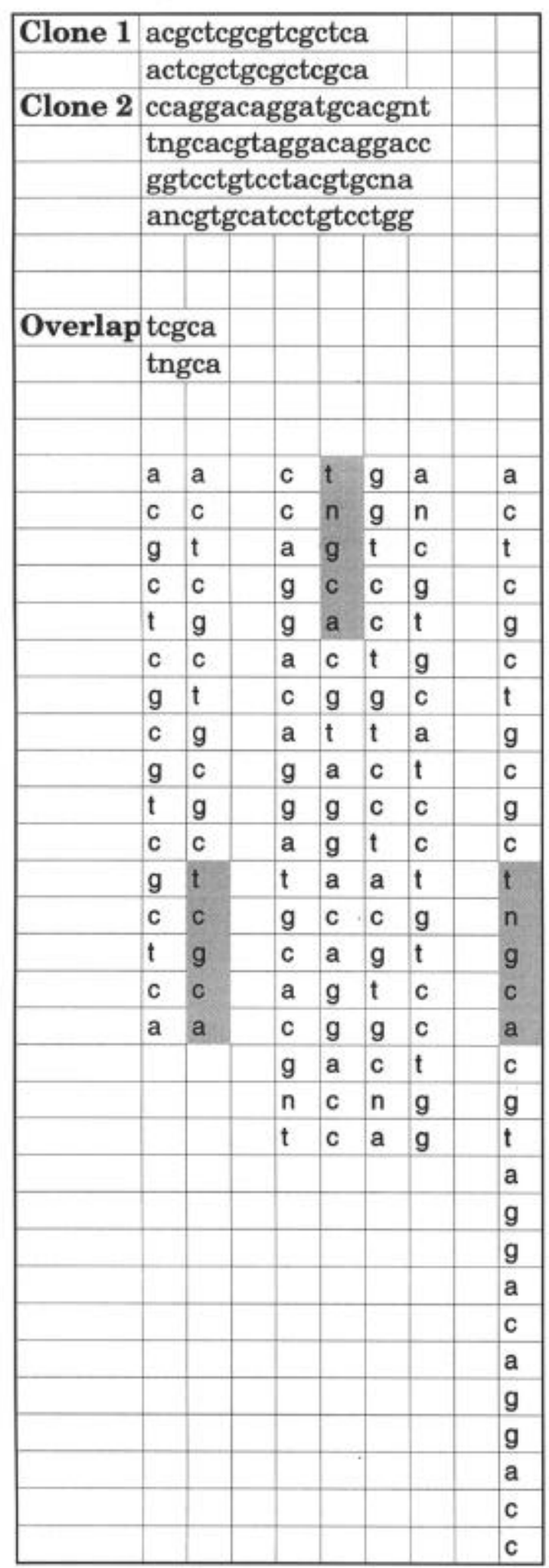

Figure 2. The result of executing the first three macros in sheet 2 . The sequences are converted into various orientations and pasted below in a vertical column format. The aligned regions are highlighted, and the alignment is displayed.

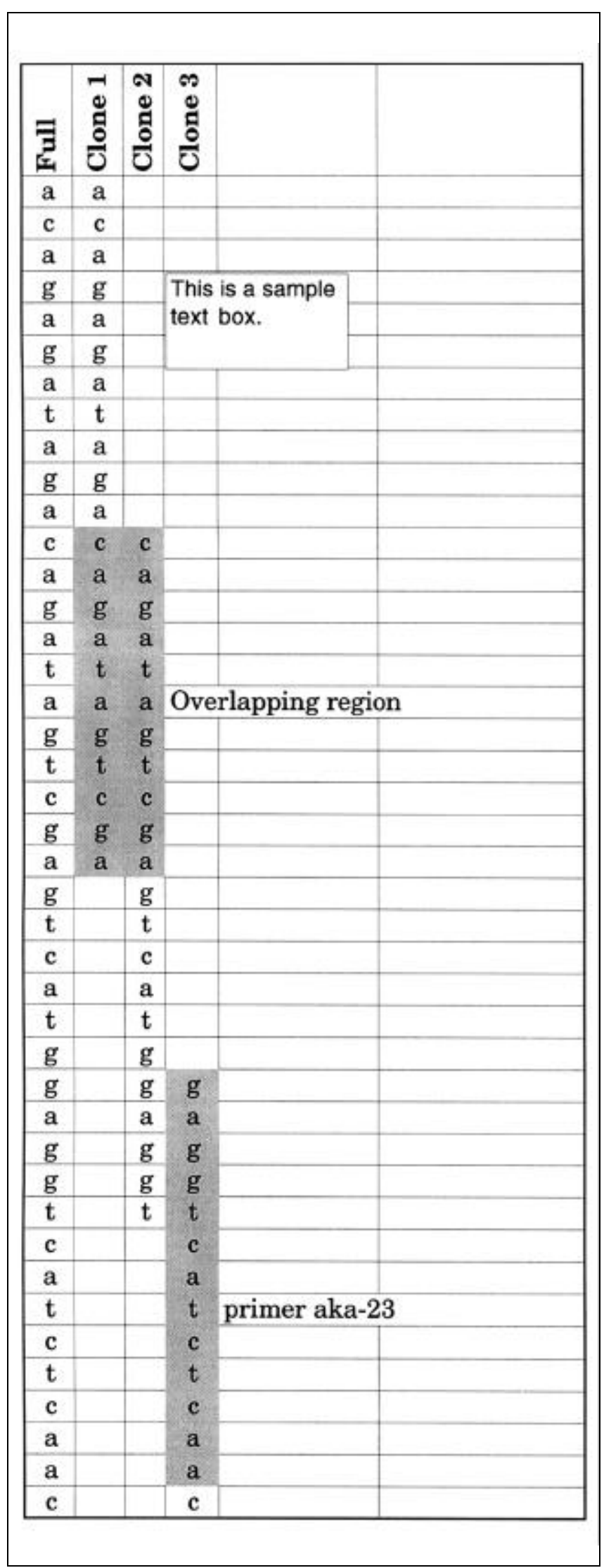

Figure 3. A sample of sheet 3 with arranged sequences. 
happen when there are internal sequences similar to the terminal region, or when the overlapping region identified has only few or a single nucleotide. If this happens, the program displays the message "Multiple matching regions found. Alignment display not performed" and does not proceed with the display of the alignment. However, all the regions that match with the overlapping sequence will be highlighted.

\section{Arranging the Overlapping Fragments into a Single Continuous Sequence}

Although the program identifies the overlapping sequence and also displays the alignment, the actual arrangement of large number of sequence fragments into a single continuous sequence has to be performed manually by the user on sheet 3 , as shown in Figure 3. To arrange a continuous sequence, fragment sequences on sheet 2, with highlighted aligned regions, are copied and pasted into sheet 3 manually, with overlapping regions aligned next to one another to show continuity. The first column of sheet 3 is allocated for entering the full continuous sequence, which can also be copied from sheet 2 . The clone and primer details are entered in the top cell of each column to keep track of the sequence identities (Figure 3). The third sheet is custom-designed to make it easy to arrange and view the sequences. The program automatically freezes the first column and top rows. If the viewing field is moved in a vertical or horizontal direction, the first column and the top row always remain visible, thus making it easy to follow up the continuity and the identity of the sequences. You can rename and save the workbooks, but the worksheet names should not be altered. We recommend making multiple copies of the downloaded file and using separate files for individual projects. Always keep a copy of the original file for future use.

\section{Other Special Functions}

The menu item Single-Step combines the functions of the three menu items (Convert, Paste and Mark-Align) and executes them in the same order in a single step. Although the entire program for aligning sequences can be run as a single step, using the three menu items individually allows the program to be used for other purposes. For example, the menu items Paste and Mark-Align could be used to search for any sequence of interest such as restriction enzyme cutting sites or $\mathrm{CpG}$ islands within a given target sequence. For these purposes, enter one or more target sequences in the cells from B1 to B6 in sheet 2. If more than one target sequence is used, enter them in the 
adjacent cells. Enter the sequence to be searched in cell B9. Now execute Paste and Mark-Align. The target sequence is pasted in a column format, and the matching regions (to the search sequence entered in cell B9) is highlighted.

While performing sequence alignments, very rarely one might face a situation in which a short fragment is contained within a longer sequence. Because the Mark-Align procedure of the program only recognizes overlapping sequence at the ends of sequences, it is not possible to identify this situation. However, if this possibility is suspected, then the following procedure can be used to identify this. Copy the longer sequence to cell B1, and paste the suspected segment in cell B9. Now, if the menu items Paste and Mark-Align are executed, the shorter segment within the longer sequence is identified.

\section{DISCUSSION}

The menu items Paste and Mark-Align are designed to aid the assembly of sequences on sheet 3 in a column format. We chose the column format for arranging sequences because the number of cells available in a column is much greater than the number available in a row. Excel 97 has 65536 cells in a column. Thus, it is possible to arrange $65 \mathrm{~kb}$ of sequence using a column format.

In addition to assembling and identifying the continuity of the sequence, a number of additional data can be recorded in sheet 3 . For example, the position of primers can be conveniently marked by color highlighting the appropriate cells, and the name of the primer can be recorded in the adjacent cell. The $5^{\prime}$ and $3^{\prime}$ ends of the sequencing primers also can be marked as indicated. Notes can be inserted to record additional information (Figure 3).

The two menu items, Paste and Mark-Align, can also be used to search for and mark amino acid sequences within a given protein sequence. If amino acid and protein sequences (entered in single letter format) are used in place of DNA sequence in the above example, the menu items Paste and Mark-Align will search and highlight the matching amino acid sequence within a target protein sequence.

The commonly available spreadsheet program Excel has been successfully used in molecular biology for a number of purposes. Shaw (3) described a method of using a spreadsheet program for protein sequence interpretation. McEwan and Gatherer (2) have described adapting a spreadsheet program to translate nucleic acid sequence into a protein sequence and to produce a codon usage table. Han and Tashjian (1) have developed a program to analyze regional hydrophobicity of a protein from its amino acid sequence. We have now described a method of using a spreadsheet program for sequence alignment. In addition to assembling a continuous sequence from small sequences of variable orientation, the program can also be used for a number of other individual needs.

\section{REFERENCES}

1.Han, B. and A.H. Tashjian, Jr. 1998. User-friendly and versatile software for analysis of protein hydrophobicity. BioTechniques 25:256-263.
2.McEwan, N.R. and D. Gatherer. 1998. Adaptation of standard spreadsheet software for the analysis of DNA sequences. BioTechniques 24:131138.

3.Shaw, G. 1995. Protein sequence interpretation using a spreadsheet program. BioTechniques 19:978-983.

Received 23 December 1998; accepted 5 April 1999.

\section{Address correspondence to:}

Dr. Ramaswamy Anbazhagan

Alpha Center/Pathology Research

5210 Eastern Avenue

Baltimore, MD 21224, USA

Internet:anba@welchlink.welch.jhu.edu 